\title{
Combination therapy with sivelestat and recombinant human soluble thrombomodulin for ARDS and DIC patients
}

This article was published in the following Dove Press journal:

Drug Design, Development and Therapy

2 September 2014

Number of times this article has been viewed

\author{
Seigo Miyoshi' \\ Ryoji Ito' \\ Hitoshi Katayama' \\ Kentaro Dote ${ }^{2}$ \\ Mayuki Aibiki ${ }^{3}$ \\ Hironobu Hamada ${ }^{1,4}$ \\ Takafumi Okura' \\ Jitsuo Higaki' \\ 'Department of Cardiology, \\ Pulmonology, Hypertension and \\ Nephrology, Ehime University \\ Graduate School of Medicine, \\ ${ }^{2}$ Intensive Care Division, Ehime \\ University Hospital, ${ }^{3}$ Department \\ of Emergency and Critical Care \\ Medicine, School of Medicine, Ehime \\ University, Shitsukawa, Toon, Ehime, \\ ${ }^{4}$ Department of Physical Analysis \\ and Therapeutic Sciences, Graduate \\ School of Biomedical and Health \\ Sciences, Hiroshima University, \\ Kasumi, Minami-ku, Hiroshima, Japan
}

Correspondence: Ryoji Ito

Department of Cardiology, Pulmonology, Hypertension and Nephrology, Ehime University Graduate School of Medicine, Shitsukawa, Toon, Ehime 79I-0295, Japan Tel +8 I 899605303

Fax +8I 899605306

Email ryito@m.ehime-u.ac.jp
Background: Neutrophil elastase, alveolar thrombin generation, and fibrin deposition play crucial roles in the development of acute respiratory distress syndrome (ARDS) and disseminated intravascular coagulation (DIC). However, the usefulness of combination therapy with a selective neutrophil elastase inhibitor, sivelestat, and recombinant human soluble thrombomodulin (rhTM) for patients with ARDS and DIC remains unknown.

Methods: We conducted a retrospective data analysis of 142 ARDS patients with DIC to assess the effects of sivelestat combined with rhTM. Patients were divided into four groups: control (no sivelestat or rhTM treatment), sivelestat treatment alone, rhTM treatment alone, and combined treatment with sivelestat and rhTM. A Cox proportional hazard model was used to assess subject mortality rates. The efficacy of these drugs was evaluated based on survival rate, number of ventilator-free days, and change in $\mathrm{PaO}_{2} / \mathrm{F}_{\mathrm{I}} \mathrm{O}_{2}(\mathrm{P} / \mathrm{F})$ ratios and DIC scores before and at 7 days after a diagnosis of ARDS with DIC.

Results: Multivariate analysis showed that patient age, combination therapy, gas exchange, organ failure, cause, associated disease score, and serum C-reactive protein levels were predictors of mortality for patients with ARDS and DIC. As compared with untreated controls, combination therapy significantly improved the 60-day survival rate of patients with ARDS and DIC. There were significantly more ventilator-free days for those who received combination therapy than for untreated controls. P/F ratios and DIC scores were significantly improved with sivelestat alone, rhTM alone, or their combination as compared with untreated controls.

Conclusion: Our results suggest that combined treatment with sivelestat and rhTM has beneficial effects on survival and the respiratory and DIC status of patients with ARDS and DIC.

Keywords: sivelestat, recombinant human soluble thrombomodulin, disseminated intravascular coagulation, acute respiratory distress syndrome

\section{Introduction}

The American-European Consensus Conference on acute respiratory distress syndrome (ARDS) defined it as a syndrome of inflammation and increased pulmonary vascular permeability. ${ }^{1}$ The pathogenesis of ARDS involves inflammatory reactions associated with the accumulation of neutrophils in the lungs. ${ }^{2-6}$ Neutrophils and neutrophil elastase, which is released from activated neutrophils, play important roles in the endothelial injury and increased permeability associated with ARDS. ${ }^{7,8}$

Disseminated intravascular coagulation (DIC) involves pathological microthrombus formation that is followed by thrombolysis in the systemic circulation, which results in the consumption of coagulation factors and platelets. ${ }^{9-12}$ Microthrombus formation causes organ ischemia, which often leads to organ failure. The rate of complications 
of ARDS and DIC gradually increases in proportion to progression of the severity of systemic inflammatory response syndrome (SIRS). ${ }^{13}$

Sivelestat (Ono Pharmaceutical Co, Ltd, Osaka, Japan) is a selective neutrophil elastase inhibitor ${ }^{14}$ that has been reported to be effective for endotoxin-induced lung injury in hamsters, guinea pigs, and sheep. ${ }^{14,15}$ Phase III and Phase IV studies conducted in Japan showed that sivelestat reduced the duration of mechanical ventilation, shortened intensive care unit stays, and prolonged the survival of patients with acute lung injury. ${ }^{16,17}$ Hayakawa et al also reported that sivelestat administration was an independent predictor for the survival of septic patients with both ARDS and DIC. ${ }^{18}$

Recombinant human soluble thrombomodulin (rhTM) suppresses thrombus formation by inhibiting thrombin coagulation activity and by activating protein $\mathrm{C}$ in complex with thrombin. ${ }^{19-21}$ rhTM administration was reportedly more effective for improvements in DIC as compared with administering heparin to patients with infection-induced DIC and hematological malignancy-associated DIC. ${ }^{22}$ Ogawa et al also reported that rhTM administration may have a significant beneficial effect on respiratory dysfunction in patients with infection-induced DIC who required ventilator management. ${ }^{23}$

Previous reports have shown the effectiveness of sivelestat for patients with ARDS and rhTM for patients with DIC. ${ }^{16-18,22,23}$ However, the usefulness of combination therapy with sivelestat and rhTM for patients with ARDS and DIC has not been determined, and only a few studies have evaluated the efficacy of sivelestat for DIC and the efficacy of rhTM for respiratory status. Thus, the aim of this study was to assess the efficacy of combination therapy with sivelestat and rhTM and whether sivelestat was useful for DIC status and rhTM was useful for the respiratory status of patients with ARDS and DIC.

\section{Materials and methods}

\section{Study population}

This was a retrospective study of ARDS patients with DIC who were admitted to Ehime University Hospital during the period from 2009 to 2013. Patients were excluded if they were younger than 20 years of age; had a neuromuscular disease that impaired spontaneous ventilation; or had severe chronic pulmonary disease, severe central nervous system disease, uncontrolled malignancy, or severe chronic liver disease. Our study protocol was approved by the local ethics committee, and informed consent was waived because of this study's retrospective design.

\section{Diagnoses of ARDS, DIC, SIRS, and sepsis}

The four criteria used for a diagnosis of ARDS were based on the Berlin definition: ${ }^{24}$ acute respiratory failure not fully explained by cardiac failure or fluid overload, bilateral opacities consistent with pulmonary edema on chest radiograph or computed tomography scan, onset within one week after a known clinical insult or new/worsening respiratory symptoms, and oxygenation with a $\mathrm{PaO}_{2} / \mathrm{F}_{\mathrm{I}} \mathrm{O}_{2}(\mathrm{P} / \mathrm{F})$ ratio $\leq 300 \mathrm{mmHg}$.

A diagnosis of SIRS was confirmed by the presence of at least two of the following conditions that were originally proposed by the American College of Chest Physicians/Society of Critical Care Medicine Consensus Conference: ${ }^{25}$ body temperature $<36^{\circ} \mathrm{C}$ or $>38^{\circ} \mathrm{C}$, heart rate $>90$ beats per minute, respiratory rate $>20$ breaths/minute or $\mathrm{PaCO}_{2}<32 \mathrm{mmHg}$, and/or white blood cell count $>12,000$ cells $/ \mu \mathrm{L}$ or $<4,000$ cells $/ \mu \mathrm{L}$, or $>10 \%$ immature (band) cells.

The scoring system of the Japanese Association for Acute Medicine $^{26}$ was used for a diagnosis of DIC. The Japanese Association for Acute Medicine DIC score includes four variables: SIRS score, platelet count, prothrombin time, and fibrin/fibrinogen degradation products. Patients were diagnosed with DIC when the summed score of these four variables was $\geq 4$.

Sepsis was diagnosed based on the following criteria proposed by the Society of Critical Care Medicine/ European Society of Intensive Care Medicine/American College of Chest Physicians/American Thoracic Society/ Surgical Infection Society International Sepsis Definition Conference, ${ }^{27}$ ie, confirmed source of infection and fulfillment of SIRS criteria.

\section{Interventions and treatments}

All patients received pressure-controlled and pressuresupported mechanical ventilation with a positive endexpiratory pressure. At the time that ARDS with DIC was diagnosed, sivelestat was continuously administered intravenously at a rate of $0.2 \mathrm{mg} / \mathrm{kg} /$ hour for a maximum of 14 days. Continuous rhTM administration was also started at a dose of $0.06 \mathrm{mg} / \mathrm{kg} /$ day for a maximum of 6 consecutive days. For this retrospective study, there were no predefined protocols regarding the definite indications for sivelestat and/or rhTM. Therefore, these drugs were administered at the discretion of the attending physician.

Except for sivelestat and rhTM therapies, all patients were primarily treated according to the strategy described in the expert consensus for the treatment of DIC in Japan. ${ }^{28}$ 
In addition, septic patients were treated according to Surviving Sepsis Campaign guidelines. ${ }^{29} \mathrm{We}$ did not administer rhAPC to all patients because rhAPC has not been approved in Japan.

\section{Data acquisition}

Baseline data were acquired from patient records. Clinical data, including ARDS etiology, the number of failed organs, and values for assessment systems, which included sequential organ failure assessment (SOFA) scores, ${ }^{30}$ gas exchange, organ failure, cause, associated disease (GOCA) scores, ${ }^{31}$ SIRS scores, and DIC scores, were collected at the time that ARDS with DIC was diagnosed. Laboratory test results, including white blood cell count, platelet count, and C-reactive protein levels, were acquired at the time that ARDS with DIC was diagnosed. Patients were followed up for 60 days after a diagnosis of ARDS with DIC.

Sivelestat or rhTM efficacy was evaluated based on survival rate, number of ventilator-free days, and change in $\mathrm{P} / \mathrm{F}$ ratio $(\Delta \mathrm{P} / \mathrm{F})$ and DIC scores $(\Delta \mathrm{DIC}$ score) between before and at 7 days after a diagnosis of ARDS with DIC. Ventilatorfree days were defined as the number of days (day 1 to day 28) that a patient breathed without assistance. ${ }^{32}$

\section{Statistical analysis}

Results are given as medians and interquartile ranges. The Mann-Whitney $U$ test or Chi-square test was used to compare results between two groups. When patients were divided into four groups (untreated controls, sivelestat alone, rhTM alone, and combination treatment), Kruskal-Wallis analysis or a chi-square test was used to compare the results of these four groups. Univariate analyses by Cox proportional hazard models were used to assess the relationships between patient mortality and the following variables: sex, age, steroid administration, sivelestat or rhTM alone, combination therapy with sivelestat and rhTM, sepsis, number of failed organs, SOFA score, GOCA score, SIRS score, DIC score, P/F ratio, white blood cell count, platelet count, and C-reactive protein serum levels at the time of diagnosis with ARDS and DIC. Variables that were found to be significant by univariate analysis were taken as potential predictors of mortality and used as covariates in multivariate analysis to identify independent predictors of mortality. To assess the clinical efficacy of sivelestat or rhTM, survival was assessed using a Cox proportional hazard model with sex, age, $\mathrm{P} / \mathrm{F}$ ratio at the time of ARDS diagnosis, number of failed organs, and septic status as covariates. Hazard ratios and 95\% confidence intervals (CIs) were determined for these variables. The Kaplan-Meier method was used to estimate survival rates, and comparisons were made using log rank tests. All tests were two-tailed, and $P$-values $<0.05$ were considered significant. Statistical analysis was performed using Statistical Package for the Social Sciences for Windows version 19 software (SPSS, Inc., Chicago, IL, USA).

\section{Results \\ Patient characteristics}

A total of 594 patients with ARDS and/or DIC were initially assessed for inclusion in this study. We excluded 113 patients who did not satisfy the definition of ARDS, 86 who did not satisfy the definition of DIC, 23 who were younger than 20 years of age, 161 who had an uncontrolled malignancy, 13 who had severe chronic pulmonary disease, 40 who had severe chronic liver disease, four who had a neuromuscular disease that impaired spontaneous ventilation, and 12 who had a severe central nervous system disease. The remaining 142 patients ( 87 men and 55 women) were included in the study. The characteristics of patients with ARDS and DIC are shown in Table 1. Their median age was 70 years and most had sepsis $(70.4 \%)$.

Table I Patient characteristics

\begin{tabular}{|c|c|}
\hline Parameter & Data \\
\hline Patients & 142 \\
\hline Sex (male/female) & $87 / 55$ \\
\hline Age (yr) & $70(62-78)$ \\
\hline Administration of steroid (yes/no) & $58 / 84$ \\
\hline Administration of sivelestat (yes/no) & $57 / 85$ \\
\hline Administration of rhTM (yes/no) & $52 / 90$ \\
\hline \multicolumn{2}{|l|}{ Etiology of ARDS } \\
\hline Sepsis (yes/no) & $100 / 42$ \\
\hline Operation (yes/no) & $7 / 135$ \\
\hline Aspiration (yes/no) & $14 / 128$ \\
\hline Others (yes/no) & $2|/| 2 \mid$ \\
\hline Number of failed organs & $2(2-3)$ \\
\hline SOFA score & II (8-13) \\
\hline GOCA score & $7(5-8)$ \\
\hline SIRS score & $3(3-4)$ \\
\hline DIC score & $5(4-6)$ \\
\hline $\mathrm{PaO}_{2} / \mathrm{F}_{1} \mathrm{O}_{2}$ ratio $(\mathrm{mmHg})$ & I 76.9 (I29.8-224.0) \\
\hline WBC $\left(\times 10^{3} / \mu \mathrm{L}\right)$ & $10.4(5.5-\mid 4.2)$ \\
\hline $\operatorname{PLT}\left(\times 10^{4} / \mu \mathrm{L}\right)$ & $8.1(5.5-12.7)$ \\
\hline CRP (mg/dL) & $12.6(4.9-2 \mid .4)$ \\
\hline
\end{tabular}

Note: Results are median values (interquartile ranges in parentheses).

Abbreviations: yr, years; rhTM, recombinant human soluble thrombomodulin; ARDS, acute respiratory distress syndrome; SOFA score, sequential organ failure assessment score; GOCA score, gas exchange, organ failure, cause, associated disease score; SIRS, systemic inflammatory response syndrome; DIC, disseminated intravascular coagulation; $\mathrm{PaO}_{2} / \mathrm{F}_{1} \mathrm{O}_{2}$ ratio, oxygen partial pressure divided by fraction of inspired oxygen; WBC, white blood cell; PLT, platelet; CRP, C-reactive protein. 


\section{Predictive variables for mortality in patients with ARDS and DIC}

Univariate analyses showed that age, sivelestat therapy, rhTM therapy, combination therapy with sivelestat and rhTM, number of failed organs, SOFA score, GOCA score, platelet count, and serum C-reactive protein levels were significantly associated with the mortality of ARDS and DIC patients (Table 2). Because there was some overlap among patients between each single therapy group and the combination therapy group, we only included combination therapy as a variable for multivariate analysis. Multivariate analysis showed that age, combination therapy, GOCA score, and serum C-reactive protein levels were predictors of mortality for ARDS patients with DIC (Table 2).

\section{Characteristics of patients with and without sivelestat and/or rhTM therapy}

We divided patients into four groups based on the administration of sivelestat and/or rhTM. The characteristics of these

Table 2 The hazard ratios and 95\% confidence intervals $(\mathrm{Cl})$ for mortality based on univariate and multivariate cox analysis in patients with ARDS and DIC

\begin{tabular}{|c|c|c|c|c|}
\hline \multirow[t]{2}{*}{ Parameter } & \multicolumn{4}{|l|}{$95 \% \mathrm{Cl}$} \\
\hline & $\begin{array}{l}\text { Hazard } \\
\text { ratio }\end{array}$ & Low & High & $P$-value \\
\hline \multicolumn{5}{|l|}{ Univariate } \\
\hline Male & 0.951 & 0.571 & 1.584 & 0.846 \\
\hline Age & 1.021 & 1.002 & $1.04 \mid$ & 0.029 \\
\hline Administration of steroid & 1.229 & 0.743 & 2.034 & 0.422 \\
\hline Administration of sivelestat & 0.529 & 0.305 & 0.918 & 0.023 \\
\hline Administration of rhTM & 0.528 & 0.305 & 0.918 & 0.029 \\
\hline Combination therapy & 0.333 & 0.121 & 0.918 & 0.034 \\
\hline Sepsis & 0.699 & 0.414 & 1.179 & 0.180 \\
\hline Number of failed organs & 1.552 & 1.092 & 2.205 & 0.014 \\
\hline SOFA score & 1.165 & 1.094 & 1.240 & $<0.001$ \\
\hline GOCA score & $1.46 \mathrm{I}$ & 1.231 & 1.735 & $<0.001$ \\
\hline SIRS score & 0.787 & 0.554 & 1.116 & 0.179 \\
\hline DIC score & 1.179 & 1.000 & 1.390 & 0.051 \\
\hline $\mathrm{PaO}_{2} / \mathrm{F}_{1} \mathrm{O}_{2}$ ratio & 0.997 & 0.993 & 1.002 & 0.221 \\
\hline WBC & 1.000 & 1.000 & 1.000 & 0.778 \\
\hline PLT & 0.943 & 0.895 & 0.993 & 0.027 \\
\hline CRP & 0.95 & 0.924 & 0.976 & $<0.001$ \\
\hline \multicolumn{5}{|l|}{ Multivariate } \\
\hline Age & 1.033 & 1.013 & 1.053 & 0.001 \\
\hline Combination therapy & 0.223 & 0.077 & 0.650 & 0.006 \\
\hline Number of failed organs & 0.686 & 0.418 & 1.127 & 0.137 \\
\hline SOFA score & 1.039 & 0.938 & 1.150 & 0.463 \\
\hline GOCA score & $\mathrm{I} .444$ & 1.122 & 1.858 & 0.004 \\
\hline PLT & 0.970 & 0.919 & 1.023 & 0.260 \\
\hline CRP & 0.951 & 0.923 & 0.979 & $<0.001$ \\
\hline
\end{tabular}

Abbreviations: ARDS, acute respiratory distress syndrome; DIC, disseminated intravascular coagulation; rhTM, recombinant human soluble thrombomodulin; SOFA score, sequential organ failure assessment score; GOCA score, gas exchange, organ failure, cause, associated disease score; SIRS, systemic inflammatory response syndrome; $\mathrm{PaO}_{2} / \mathrm{F}_{1} \mathrm{O}_{2}$ ratio, oxygen partial pressure divided by fraction of inspired oxygen; WBC, white blood cell; PLT, platelet; CRP, C-reactive protein. patients at the time of their diagnosis of ARDS with DIC are shown in Table 3. There were 54 patients in the control group, 36 patients in the sivelestat alone group, 31 patients in the rhTM alone group, and 21 patients in the combination therapy group. There were no significant differences in the results for any of the variables between these four groups.

\section{Efficacy of sivelestat and/or rhTM for ARDS and DIC patients}

Kaplan-Meier survival curves are shown in Figure 1. As compared with untreated controls, combination therapy significantly improved the 60-day survival rate of patients with ARDS and DIC $(P=0.004)$. The survival rate with each single therapy alone tended to be higher than that of the control group, although these differences were not significant ( $P=0.064$ for sivelestat alone and $P=0.081$ for rhTM alone). The ventilator-free day results for the combination group were significantly better than those for the control group (Figure 2A). P/F ratios and DIC scores at 7 days after a diagnosis of ARDS with DIC significantly improved with each single therapy and the combination therapy (Figure 2B and $\mathrm{C}$ ).

\section{Patient clinical factors amenable to sivelestat and rhTM therapy}

Sivelestat administration was significantly effective for patients with a $\mathrm{P} / \mathrm{F}$ ratio $\geq 100 \mathrm{mmHg}$ and sepsis (Figure 3). By comparison, rhTM administration was effective for patients aged $\geq 65$ years, a $\mathrm{P} / \mathrm{F}$ ratio of $\geq 100 \mathrm{mmHg}$, fewer than three failed organs, and sepsis (Figure 3). In addition, combination therapy was significantly effective for patients aged $\geq 65$ years (hazard ratio [HR] 3.224; 95\% CI $1.153-9.021 ; P=0.026$ ), with a $\mathrm{P} / \mathrm{F}$ ratio of $\geq 100 \mathrm{mmHg}$ (HR 4.601; 95\% CI 1.118-18.936; $P=0.034$ ), with fewer than three failed organs (HR 4.668; 95\% CI 1.119-19.468; $P=0.034$ ), and with sepsis (HR 3.310; 95\% CI 1.020-10.743; $P=0.046)$.

\section{Adverse events}

During the study period, two adverse events involving hepatic dysfunction occurred in the sivelestat group. One serious adverse event related to bleeding (gastrointestinal hemorrhage) occurred in the rhTM group. One serious adverse event related to bleeding (gastrointestinal hemorrhage) occurred in the combination group. The incidence of adverse events was $5.6 \%(2 / 36)$ in the sivelestat group, $3.2 \%$ $(1 / 31)$ in the rhTM group, and $4.8 \%(1 / 21)$ in the combination group. The adverse events associated with sivelestat 
Table 3 Patient characteristics treated or not treated with sivelestat and/or rhTM

\begin{tabular}{|c|c|c|c|c|c|}
\hline & Control & Sivelestat & rhTM & Combination & $P$-value \\
\hline Patients & 54 & 36 & 31 & 21 & \\
\hline Sex (male/female) & $32 / 22$ & $18 / 18$ & $21 / 10$ & $16 / 5$ & 0.209 \\
\hline Age (yr) & $70(60-77)$ & $70(57-76)$ & $67(63-76)$ & $78(70-8 I)$ & 0.067 \\
\hline Administration of steroid (yes/no) & $19 / 35$ & $21 / 15$ & $11 / 20$ & $7 / 14$ & 0.105 \\
\hline \multicolumn{6}{|l|}{ Etiology of ARDS } \\
\hline Sepsis (yes/no) & $35 / 19$ & $25 / 11$ & $23 / 8$ & $17 / 4$ & 0.540 \\
\hline Operation (yes/no) & $2 / 52$ & $2 / 34$ & $3 / 28$ & $0 / 21$ & 0.426 \\
\hline Aspiration (yes/no) & $6 / 48$ & $5 / 31$ & $1 / 30$ & $2 / 19$ & 0.514 \\
\hline Others (yes/no) & $11 / 43$ & $4 / 32$ & $4 / 27$ & $2 / 19$ & 0.518 \\
\hline Number of failed organs & $2(I-3)$ & $2(I-2)$ & $2(2-3)$ & $2(1.5-2)$ & 0.100 \\
\hline SOFA score & II (8-13) & $10(8-12)$ & $12(10-14)$ & $10(8-12)$ & 0.073 \\
\hline GOCA score & $7(5-8)$ & $6(5-7)$ & $7(6-8)$ & $7(6-8)$ & 0.100 \\
\hline SIRS score & $3(3-4)$ & $3(3-3)$ & $3(2-4)$ & $3(3-4)$ & 0.783 \\
\hline DIC score & $4(4-5)$ & $4(4-7)$ & $5(5-7)$ & $5(4-5)$ & 0.230 \\
\hline $\mathrm{PaO}_{2} / \mathrm{F}_{1} \mathrm{O}_{2}$ ratio $(\mathrm{mmHg})$ & $190.2(\mid 50.0-238.1)$ & I50.I (I |6.5-22I.4) & | 87.7 (| 58.2-225.0) & I 38.8 (95. I-208.7) & 0.083 \\
\hline $\mathrm{WBC}\left(\times 10^{3} / \mu \mathrm{L}\right)$ & $11.9(7.2-14.5)$ & $10.3(6.4-14.0)$ & $7.3(4.2-11.7)$ & $8.1(5|-| 7.7)$ & 0.118 \\
\hline $\operatorname{PLT}\left(\times 10^{4} / \mu \mathrm{L}\right)$ & $10.0(5.9-12.7)$ & $9.0(5.7-15.7)$ & $6.6(4.4-9.3)$ & $7.7(5.6-14.6)$ & 0.102 \\
\hline CRP (mg/dL) & $10.9(4.3-20.8)$ & I4.8 (6.7-|8.5) & $12.6(4 . \mid-25.4)$ & I $7.4(7.8-29.0)$ & 0.585 \\
\hline
\end{tabular}

Note: Results are median values (interquartile ranges in parentheses).

Abbreviations: yr, years; rhTM, recombinant human soluble thrombomodulin; ARDS, acute respiratory distress syndrome; SOFA score, sequential organ failure assessment score; GOCA score, gas exchange, organ failure, cause, associated disease score; SIRS, systemic inflammatory response syndrome; DIC, disseminated intravascular coagulation; $\mathrm{PaO}_{2} / \mathrm{F}_{1} \mathrm{O}_{2}$ ratio, oxygen partial pressure divided by fraction of inspired oxygen; WBC, white blood cell; PLT, platelet; CRP, C-reactive protein.

use improved after discontinuing the drug. Serious adverse events associated with rhTM use recovered using hemostatic therapy.

\section{Discussion}

In this study, we demonstrated that treatment with both sivelestat and rhTM could significantly prolong the survival of patients with ARDS and DIC and that a decision whether or not to administer both sivelestat and rhTM might be associated with the mortality of patients with ARDS and DIC. In addition, each single therapy regimen and the combination

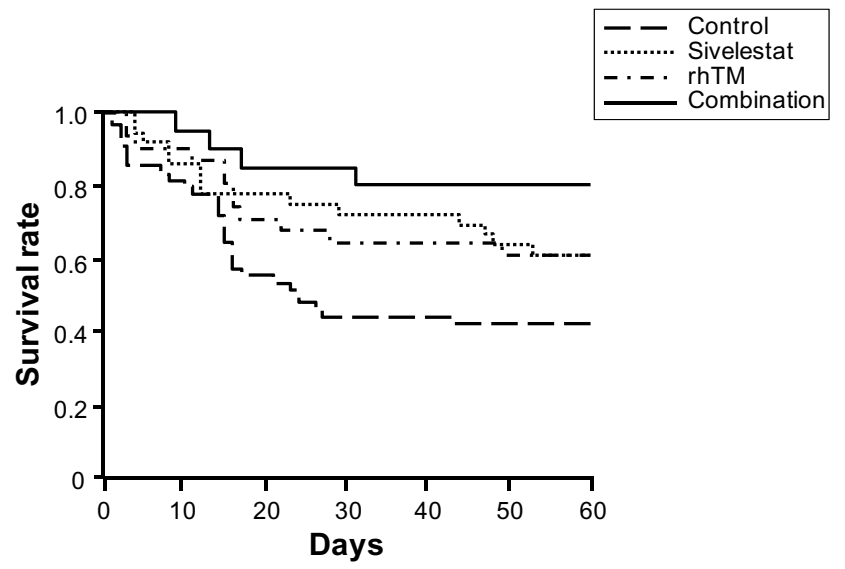

Figure I Kaplan-Meier survival curves for acute respiratory distress syndrome patients with disseminated intravascular coagulation who did or did not receive sivelestat and/or rhTM. Statistical comparisons were made by log rank tests. Abbreviation: rhTM, recombinant human soluble thrombomodulin. therapy resulted in improved respiratory and DIC status in ARDS and DIC patients. Thus, we propose that administering sivelestat and rhTM may be useful for patients with ARDS and DIC.

Treatment with sivelestat or rhTM may improve the respiratory status of ARDS patients with DIC. Neutrophils and neutrophil elastase are thought to play critical roles in the endothelial injury and increased permeability involved in the pathogenesis of ARDS. ${ }^{7,8}$ Sivelestat is a selective neutrophil elastase inhibitor and it was reported that administering sivelestat improved ventilator-free day results and ventilator weaning rates in patients with acute lung injury and SIRS. ${ }^{17}$ Our study also showed that treatment with sivelestat significantly improved $\mathrm{P} / \mathrm{F}$ ratios at 7 days after a diagnosis of ARDS with DIC as compared with the untreated control group.

Regarding rhTM therapy, it was reported that rhTM suppressed thrombus formation by inhibiting thrombin coagulation activity and by activating protein $\mathrm{C}$ in complex with thrombin, ${ }^{19-21}$ and has been generally used for DIC treatment. It was also reported that protein $\mathrm{C}$ prevented an increase in endotoxin-induced pulmonary vascular permeability by inhibiting tumor necrosis factor- $\alpha$ production in rats. ${ }^{33}$ Uchiba et al reported that rhTM prevented endotoxin-induced pulmonary vascular injury by inhibiting pulmonary accumulation of leukocytes mediated through thrombin binding and subsequent protein $\mathrm{C}$ activation. ${ }^{34}$ Ogawa et al also reported 
A

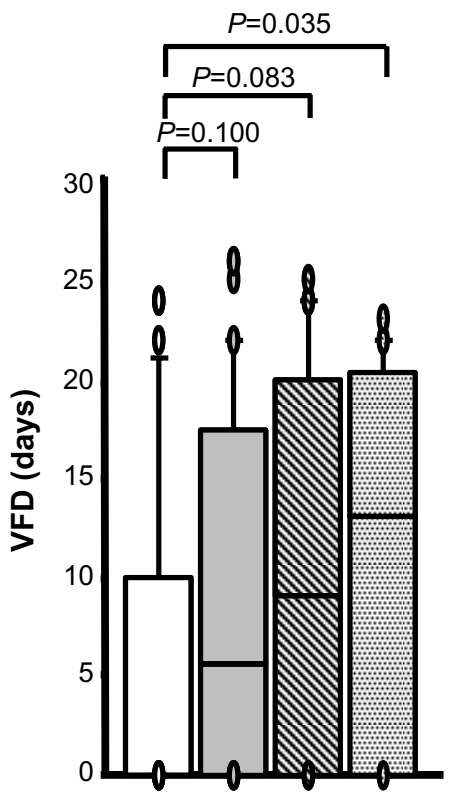

B

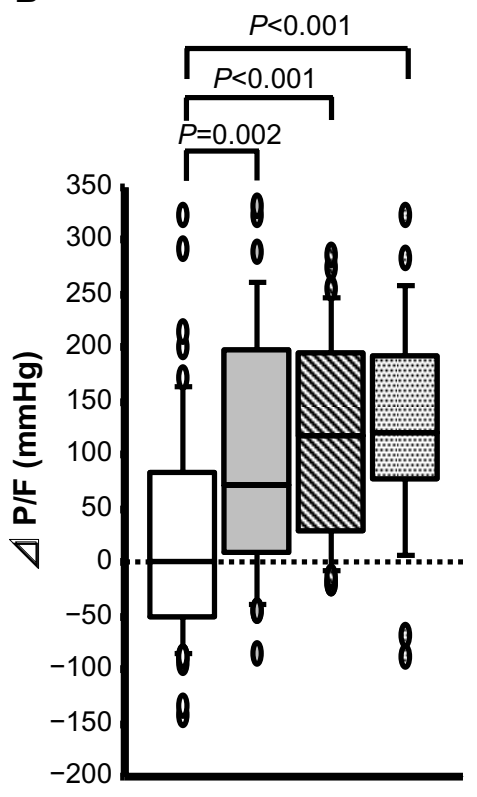

C

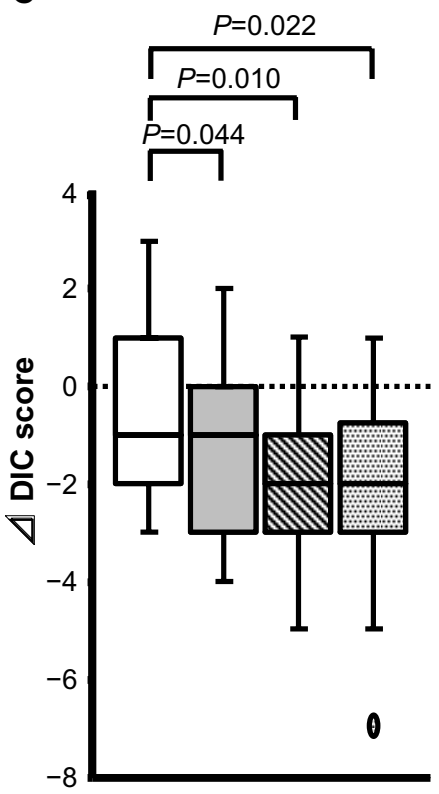

Figure 2 Clinical efficacies of sivelestat, rhTM, and combination therapy. Efficacy assessments were made based on (A) VFD, (B) $\Delta \mathrm{P} / \mathrm{F}$, and (C) $\Delta \mathrm{DIC}$ score between before and at 7 days after a diagnosis of acute respiratory distress syndrome with DIC. Statistical comparisons were made using the Mann-Whitney $U$ test.

Abbreviations: rhTM, recombinant human soluble thrombomodulin; VFD, ventilator-free days; $\Delta \mathrm{DIC}$, changes in disseminated intravascular coagulation; $\Delta \mathrm{P} / \mathrm{F}$, change in $\mathrm{PaO}_{2} / \mathrm{F}_{1} \mathrm{O}_{2}$ ratio; $\mathrm{PaO}_{2} / \mathrm{F}_{1} \mathrm{O}_{2}$ ratio, oxygen partial pressure divided by fraction of inspired oxygen.

that the respiratory component of the SOFA score and the lung injury score in the rhTM group significantly decreased as compared with those in the control group..$^{23}$ Our results also showed that rhTM administration significantly improved $\mathrm{P} / \mathrm{F}$ ratios at 7 days after a diagnosis of ARDS with DIC as compared with the control group. These results suggest that sivelestat and rhTM may be useful for improving the respiratory status of patients with ARDS and DIC.

Treatment with sivelestat or rhTM may also be useful for improving the DIC status of patients with ARDS and DIC.

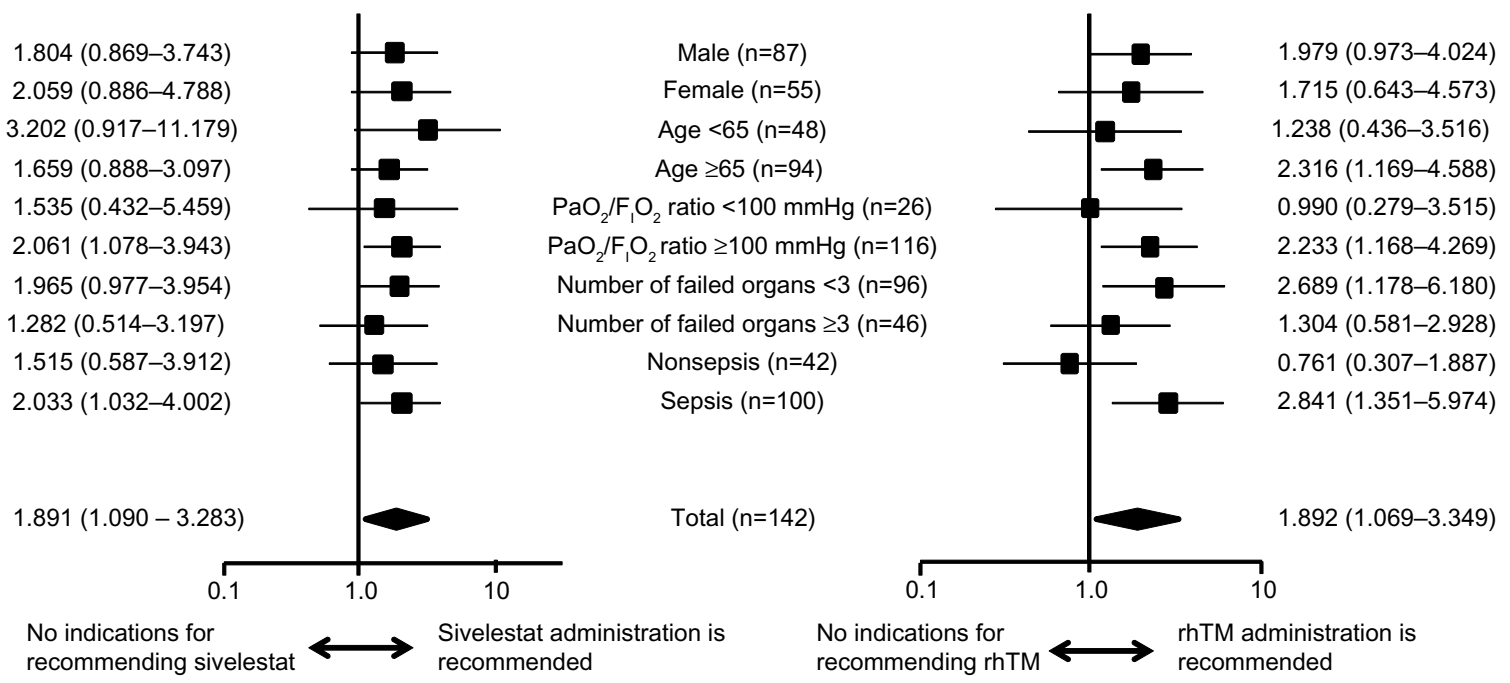

Figure 3 Hazard ratios and 95\% confidence intervals for survival based on Cox proportional hazard models for acute respiratory distress syndrome patients with disseminated intravascular coagulation.

Abbreviation: rhTM, recombinant human soluble thrombomodulin; $\mathrm{PaO}_{2} / \mathrm{F}_{1} \mathrm{O}_{2}$ ratio, oxygen partial pressure divided by fraction of inspired oxygen. 
It was reported that neutrophils and neutrophil elastase play critical roles in the pathogenesis of DIC. ${ }^{35}$ Systemic inflammation characterized by excessive production of proinflammatory cytokines activates neutrophils and induces their excessive release of neutrophil elastase. ${ }^{36-38}$ This excess neutrophil elastase and other proinflammatory mediators synergistically injure endothelial cells. ${ }^{36-38}$ Tissue factor is expressed on injured endothelial cells and activated mononuclear cells. ${ }^{9,35,39,40}$ This tissue factor triggers the procoagulant cascade, induces aberrant coagulation and fibrinolysis, and results in DIC. Hayakawa et al reported that the time until DIC scores improved was shorter in a sivelestat group than in a control group. ${ }^{18}$ Our study also showed that the improvement in DIC scores at 7 days after a diagnosis of ARDS with DIC was significantly greater in the sivelestat group than in the control group.

Regarding rhTM efficacy, Yamakawa et al reported that both Japanese Association for Acute Medicine and International Society of Thrombosis and Hemostasis DIC scores in the rhTM group were significantly decreased as compared with those in the control group. ${ }^{41}$ In addition, Saito et al reported that rhTM therapy was significantly superior to heparin administration for improving DIC status. ${ }^{22}$ In the present study, we also demonstrated that $\triangle \mathrm{DIC}$ scores in the rhTM group significantly decreased as compared with those in the control group. These results suggested that sivelestat or rhTM therapy may be useful for improving DIC status in patients with ARDS and DIC.

Combination therapy with sivelestat and rhTM may be useful and reasonable for patients with ARDS and DIC. As noted above, the present study demonstrated that each single therapy regimen may improve respiratory and DIC status in patients with ARDS and DIC. This combination therapy showed results that were similar to those achieved with each single therapy regimen. In addition, our results showed that the combination therapy significantly improved the 60-day survival rate in patients with ARDS and DIC. The ventilator-free day results in the combination therapy group were significantly better than those in the control group. Multivariate analysis showed that a decision whether or not to administer both sivelestat and rhTM was significantly associated with the mortality of patients with ARDS and DIC. These result suggested that combined treatment with sivelestat and rhTM might have beneficial effects for ARDS patients with DIC.

Age, respiratory condition, septic status, and the number of failed organs might have affected the efficacy of the combination therapy in patients with ARDS and DIC. In our previous study, we showed that baseline respiratory function and septic status might have affected the efficacy of sivelestat use for patients with acute lung injury and SIRS. ${ }^{42}$ It was also reported that rhTM may have a significant beneficial effect on mortality and respiratory dysfunction in patients with sepsis-induced DIC. ${ }^{19,23}$ Our present study results were in agreement with those in these previous reports. Additionally, in the present study, combination therapy was significantly more effective for patients aged $\geq 65$ years and those in whom the number of failed organs was less than three. Further studies will be needed to clarify the clinical factors that are favorable for using this combination therapy in patients with ARDS and DIC.

Unlike similar previous studies, we did not obtain statistically significant differences in survival and ventilator-free days between each single therapy group and the control group. This might be related to differences in patient characteristics. Previous studies included DIC patients with sepsis, whereas our study included heterogeneous groups of DIC patients with ARDS induced by diseases other than sepsis. In addition, we previously reported that sivelestat might be beneficial for patients with acute lung injury and sepsis, but not for patients with acute lung injury without sepsis. ${ }^{42}$ In the present study, we also demonstrated that administration of sivelestat and rhTM was more effective for patients with sepsis than for those without sepsis (Figure 3).

Sivelestat and rhTM therapy might be tolerated by patients with ARDS and DIC. Previous reports indicated that the frequency of adverse events in sivelestat or rhTM groups was not higher than that in control groups. ${ }^{17,22,23}$ The incidence of adverse events with sivelestat use in the present study was lower than that in a previous report. ${ }^{17}$ In addition, the incidence of adverse events with rhTM use in the present study was similar to that in a previous report. ${ }^{22}$ These results indicate that sivelestat and rhTM toxicities are acceptable for patients with ARDS and DIC.

The limitations of this study include the following. Our study was a retrospective analysis and the number of patients was small. We made no restrictions on the use of other drugs in addition to sivelestat and rhTM, and we did not evaluate other therapies except for the use of steroids.

In conclusion, our study results demonstrate that the combination of sivelestat and rhTM may improve the survival of patients with ARDS and DIC. Each single therapy alone and the combination of sivelestat and rhTM may be useful for improving the respiratory and DIC status of patients with ARDS and DIC. However, a large prospective study 
is needed to establish the usefulness of sivelestat and rhTM for ARDS patients with DIC.

\section{Disclosure}

JH has received research grants from Takeda Pharm Ltd, Mochida Pharm Ltd, Novartis Pharm Ltd, Pfizer Ltd, MSD Ltd, Astellas Pharm Ltd, Japan Boehringer-Ingelheim Ltd, Daiichi-Sankyo Ltd, and Dainihon-Sumitomo Pharm Ltd, and has received honoraria from Takeda Pharm Ltd, InterScience Ltd, Mochida Pharm Ltd, Novartis Pharm Ltd, Pfizer Ltd, MSD Ltd, Astellas Pharm Ltd, Japan BoehringerIngelheim Ltd, Dainihon-Sumitomo Pharm Ltd, and Teijin Pharm Ltd. The other authors report no conflicts of interest in this work.

\section{References}

1. Bernard GR, Artigas A, Brigham KL, et al. The American-European Consensus Conference on ARDS. Definitions, mechanisms, relevant outcomes, and clinical trial coordination. Am J Respir Crit Care Med. 1994;149:818-824.

2. Ware LB, Matthay MA. The acute respiratory distress syndrome. N Engl J Med. 2000;342:1334-1349.

3. Lee WL, Downey GP. Leukocyte elastase: physiological functions and role in acute lung injury. Am J Respir Crit Care Med. 2001;164: 896-904.

4. Weiland JE, Davis WB, Holter JF, Mohammed JR, Dorinsky PM, Gadek JE. Lung neutrophils in the adult respiratory distress syndrome. Clinical and pathophysiologic significance. Am Rev Respir Dis. 1986;133:218-225.

5. Idell S, Kucich U, Fein A, et al. Neutrophil elastase-releasing factors in bronchoalveolar lavage from patients with adult respiratory distress syndrome. Am Rev Respir Dis. 1985;132:1098-1105.

6. Donnelly SC, MacGregor I, Zamani A, et al. Plasma elastase levels and the development of the adult respiratory distress syndrome. Am J Respir Crit Care Med. 1995;151:1428-1433.

7. Abraham E. Neutrophils and acute lung injury. Crit Care Med. 2003;31:S195-S199.

8. Moraes TJ, Chow CW, Downey GP. Proteases and lung injury. Crit Care Med. 2003;31:S189-S194.

9. Levi M, Ten Cate H. Disseminated intravascular coagulation. $N$ Engl J Med. 1999;341:586-592.

10. Taylor FB Jr, Toh CH, Hoots WK, Wada H, Levi M. Towards definition, clinical and laboratory criteria, and a scoring system for disseminated intravascular coagulation. Thromb Haemost. 2001;86: $1327-1330$

11. Toh CH, Dennis M. Disseminated intravascular coagulation: old disease, new hope. BMJ. 2003;327:974-977.

12. Levi M, Toh CH, Thachil J, Watson HG. Guidelines for the diagnosis and management of disseminated intravascular coagulation. British Committee for Standards in Haematology. Br J Haematol. 2009;145:24-33.

13. Rangel-Frausto MS, Pittet D, Costigan M, Hwang T, Davis CS, Wenzel RP. The natural history of the systemic inflammatory response syndrome (SIRS). A prospective study. JAMA. 1995;273:117-123.

14. Kawabata K, Suzuki M, Sugitani M, Imaki K, Toda M, Miyamoto T. ONO-5046, a novel inhibitor of human neutrophil elastase. Biochem Biophys Res Commun. 1991;177:814-820.

15. Iba T, Kidokoro A, Fukunaga M, Takuhiro K, Yoshikawa S, Sugimoto K. Pretreatment of sivelestat sodium hydrate improves the lung microcirculation and alveolar damage in lipopolysaccharide-induced acute lung inflammation in hamsters. Shock. 2006;26:95-98.
16. Tamakuma S, Ogawa M, Aikawa N, et al. Relationship between neutrophil elastase and acute lung injury in humans. Pulm Pharmacol Ther. 2004; 17:271-279.

17. Aikawa N, Ishizaka A, Hirasawa H, et al. Reevaluation of the efficacy and safety of the neutrophil elastase inhibitor, Sivelestat, for the treatment of acute lung injury associated with systemic inflammatory response syndrome; a phase IV study. Pulm Pharmacol Ther. 2011:24:549-554.

18. Hayakawa M, Katabami K, Wada T, et al. Sivelestat (selective neutrophil elastase inhibitor) improves the mortality rate of sepsis associated with both acute respiratory distress syndrome and disseminated intravascular coagulation patients. Shock. 2010;33:14-18.

19. Yamakawa K, Fujimi S, Mohri T, et al. Treatment effects of recombinant human soluble thrombomodulin in patients with severe sepsis: a historical control study. Crit Care. 2011;15:R123.

20. Nakashima M, Kanamaru M, Umemura K, Tsuruta K. Pharmacokinetics and safety of a novel recombinant soluble human thrombomodulin, ART-123, in healthy male volunteers. J Clin Pharmacol. 1998;38: $40-44$.

21. Moll S, Lindley C, Pescatore S, et al. Phase I study of a novel recombinant human soluble thrombomodulin, ART-123. J Thromb Haemost. 2004;2:1745-1751.

22. Saito H, Maruyama I, Shimazaki S, et al. Efficacy and safety of recombinant human soluble thrombomodulin (ART-123) in disseminated intravascular coagulation: results of a phase III, randomized, double-blind clinical trial. J Thromb Haemost. 2007;5:31-41.

23. Ogawa Y, Yamakawa K, Ogura H, et al. Recombinant human soluble thrombomodulin improves mortality and respiratory dysfunction in patients with severe sepsis. J Trauma Acute Care Surg. 2012;72: $1150-1157$.

24. Ranieri VM, Rubenfeld GD, Thompson BT, et al; ARDS Definition Task Force. Acute respiratory distress syndrome: the Berlin definition. JAMA. 2012;307:2526-2533.

25. [No authors listed]. American College of Chest Physicians/Society of Critical Care Medicine Consensus Conference: definitions for sepsis and organ failure and guidelines for the use of innovative therapies in sepsis. Crit Care Med. 1992;20:864-874.

26. Gando S, Iba T, Eguchi Y, et al. A multicenter, prospective validation of disseminated intravascular coagulation diagnostic criteria for critically ill patients: comparing current criteria. Crit Care Med. 2006;34:625-631.

27. Levy MM, Fink MP, Marshall JC, et al. SCCM/ESICM/ACCP/ATS/SIS. $2001 \mathrm{SCCM} / \mathrm{ESICM} / \mathrm{ACCP} / \mathrm{ATS} / \mathrm{SIS}$ International Sepsis Definitions Conference. Crit Care Med. 2003;31:1250-1256.

28. Wada H, Asakura H, Okamoto K, et al. Japanese Society of Thrombosis Hemostasis/DIC subcommittee. Expert consensus for the treatment of disseminated intravascular coagulation in Japan. Thromb Res. 2010;125:6-11.

29. Dellinger RP, Levy MM, Carlet JM, et al. International Surviving Sepsis Campaign Guidelines Committee; American Association of Critical-Care Nurses; American College of Chest Physicians; American College of Emergency Physicians; Canadian Critical Care Society; European Society of Clinical Microbiology and Infectious Diseases; European Society of Intensive Care Medicine; European Respiratory Society; International Sepsis Forum; Japanese Association for Acute Medicine; Japanese Society of Intensive Care Medicine; Society of Critical Care Medicine; Society of Hospital Medicine; Surgical Infection Society; World Federation of Societies of Intensive and Critical Care Medicine. Surviving Sepsis Campaign: international guidelines for management of severe sepsis and septic shock: 2008. Crit Care Med. 2008;36:296-327.

30. Ferreira FL, Bota DP, Bross A, Mélot C, Vincent JL. Serial evaluation of the SOFA score to predict outcome in critically ill patients. JAMA. 2001;286:1754-1758.

31. Jegal Y, Lee SI, Lee KH, et al. The clinical efficacy of GOCA scoring system in patients with acute respiratory distress syndrome. $J$ Korean Med Sci. 2008;23:383-389. 
32. The Acute Respiratory Distress Syndrome Network. Ventilation with lower tidal volumes as compared with traditional tidal volumes for acute lung injury and the acute respiratory distress syndrome. $N$ Engl J Med. 2000;342:1301-1308.

33. Murakami K, Okajima K, Uchiba M, et al. Activated protein C prevents LPS-induced pulmonary vascular injury by inhibiting cytokine production. Am J Physiol. 1997;272:L197-L202.

34. Uchiba M, Okajima K, Murakami K, et al. rhs-TM prevents ETinduced increase in pulmonary vascular permeability through protein C activation. Am J Physiol. 1997;273:L889-L894.

35. Slofstra SH, Spek CA, ten Cate H. Disseminated intravascular coagulation. Hematol J. 2003;4:295-302.

36. Russell JA. Management of sepsis. N Engl J Med. 2006;355: 1699-1713.

37. Ueno H, Hirasawa H, Oda S, Shiga H, Nakanishi K, Matsuda K Coagulation/fibrinolysis abnormality and vascular endothelial damage in the pathogenesis of thrombocytopenic multiple organ failure. Crit Care Med. 2002;30:2242-2248.
38. McGill SN, Ahmed NA, Christou NV. Endothelial cells: role in infection and inflammation. World J Surg. 1998;22:171-178.

39. Ten Cate H. Pathophysiology of disseminated intravascular coagulation in sepsis. Crit Care Med. 2000;28:S9-S11.

40. Levi M. Disseminated intravascular coagulation. Crit Care Med. 2007;35:2191-2195.

41. Yamakawa K, Ogura H, Fujimi S, et al. Recombinant human soluble thrombomodulin in sepsis-induced disseminated intravascular coagulation: a multicenter propensity score analysis. Intensive Care Med. 2013;39:644-652.

42. Miyoshi S, Hamada H, Ito R, et al. Usefulness of a selective neutrophil elastase inhibitor, sivelestat, in acute lung injury patients with sepsis. Drug Des Devel Ther. 2013;7:305-316.

\section{Publish your work in this journal}

Drug Design, Development and Therapy is an international, peerreviewed open-access journal that spans the spectrum of drug design and development through to clinical applications. Clinical outcomes, patient safety, and programs for the development and effective, safe, and sustained use of medicines are a feature of the journal, which has also been accepted for indexing on PubMed Central. The manuscript management system is completely online and includes a very quick and fair peer-review system, which is all easy to use. Visit http://www.dovepress.com/testimonials.php to read real quotes from published authors.

Submit your manuscript here: http://www.dovepress.com/drug-design-development-and-therapy-journal 